\title{
Recovery of the PRE following ECS
}

\author{
A. GRANT YOUNG and G. L. DEMPSEY \\ Louisiana State University, Baton Rouge, Louisiana 70803
}

\begin{abstract}
Ninety-six male albino rats were trained to leverpress for sucrose on either a continuous reinforcement schedule (CRF) or a variable ratio schedule of reinforcement (VR). Following acquisition, a leverpress produced footshock followed by ECS for half of the subjects in each group. For the remaining subjects, a leverpress produced footshock only. All subjects were then tested in an extinction session either 4,24 , or $48 \mathrm{~h}$ after treatment. Results showed an insignificant PRE for subjects tested 4 or $24 \mathrm{~h}$ after treatment; however, when the ECS-test interval was increased to $48 \mathrm{~h}$ there was apparent spontaneous recovery of the PRE.
\end{abstract}

When electroconvulsive shock (ECS) is administered shortly after a learning trial, the results appear to be a retrograde amnesia (RA) for that learning event (Lewis, 1969). This retrograde effect of ECS on learned responses has been accounted for by a concept of memory consolidation, which suggests that the fixation of a memory trace occurs over a period of time (McGaugh, 1966 .) During this time, it is assumed that some type of short-term process maintains the memory trace until a permanent structural change can be effected. It is during this fixation period that the massive electrical interference of ECS presumably disrupts the consolidation of the ongoing memory trace and produces RA.

A typical procedure for the study of ECS-induced RA is to administer an aversive stimulus, such as footshock, after the occurrence of a previously learned response. The aversive stimulation results in a suppression of the previously learned response; however, if the aversive stimulus is followed shortly thereafter by ECS, response suppression does not occur and RA apparently results for that one-trial learning situation.

Recently Young and Galluscio (1970) reported findings that do not appear to support a memory consoidation hypothesis. Under both a continuous reinforcement schedule (CRF) and a fixed ratio schedule of reinforcement (FR), subjects were subjected to either footshock only or footshock plus ECS, and then were tested in extinction $24 \mathrm{~h}$ later. As expected, they found that ECS apparently produced RA in the group trained on CRF; however ECS did not appear to produce RA in the FR-trained subject. It is not at all clear that a memory consolidation hypothesis would predict these results.

Additionally, a number of studies have indicated that the effects of ECS may be transient in nature, resulting in apparent spontaneous recovery from ECS-produced amnesia (Cooper \& Koppenaal, 1964; Kohlenbert \& Trabasso, 1968). Presumably, if ECS is administered while the memory trace is labile, during the fixation

This research was supported in part by a grant from the University Council on Research, LSU, to the first author. period, then the loss of that memory trace should be permanent.

Young and Galluscio (1971) trained two groups of rats to leverpress, one group on CRF and the other on a FR reinforcement schedule. Following acquisition, a leverpress produced footshock followed by ECS for half of the subjects in each group, and for the remaining subjects a leverpress produced footshock only. All subjects were tested in extinction either $24 \mathrm{~h}$ after treatment, or after a 10-day delay period. Results showed that for subjects tested 10 days after treatment, there was apparent spontaneous recovery of the conditioned emotional response resulting in no differences between the shock only and shock plus ECS groups. This finding was due to a decline in responding for the delayed-ECS groups, which made significantly fewer responses than did the ECS groups experiencing no delay. Consistent with earlier findings FR-trained subjects failed to show the PRE, and there was no apparent recovery of the PRE following the 10-day delay period.

The present study was designed to determine if the effects of ECS following training on a variable ratio schedule of reinforcement (VR) are transient, and also whether or not VR-trained subjects will show spontaneous recovery of the PRE.

\section{METHOD}

\section{Subjects}

The subjects were 96 male albino rats, approximately $200 \mathrm{~g}$ in weight at the start of the experiment.

\section{Apparatus}

The apparatus consisted of two identical Lehigh Valley operant chambers, each enclosed in a sound-insulated, ventilated cubicle. Each operant chamber had a grid floor, and fitted on the end wall was a retractable lever and a liquid dipper which dispensed $.01 \mathrm{ml}$ of a $40 \%$ sucrose solution used as reinforcement. All experimenter-controlled events were operated by an electronic programming device.

\section{Procedure}

The subjects were selected randomly from the LSU colony, placed in individual cages and kept on an ad-lib food and water 
Table 1

Mean Number of Leverpresses in Extinction

\begin{tabular}{llllll}
\hline Group & Total & Group & Total & Group & Total \\
\hline CR(ECS)4 & 53.38 & CR(ECS)24 & 52.75 & CR(ECS)48 & 52.00 \\
VR(ECS)4 & 61.63 & VR(ECS)24 & 68.75 & VR(ECS)48 & 73.00 \\
CR(SO)4 & 30.88 & CR(SO)24 & 22.50 & CR(SO)48 & 33.00 \\
VR(SO)4 & 37.88 & VR(SO)24 & 22.50 & VR(SO)48 & 34.88 \\
\hline
\end{tabular}

schedule for 4 day's, after which subjects were placed on a food deprivation schedule of $10 \mathrm{~g}$ of Purina Chow every $24 \mathrm{~h}$. Water was available in the cages at all times, and subjects were fed approximately $10 \mathrm{~min}$ after each experimental session.

From the 5th to the 9 th day, subjects were handled in pairs for $5 \mathrm{~min}$ daily, and on the 10 th day, subjects began magazine training on a VI $30-\mathrm{sec}$ schedule. Experimental periods consisted of 20 presentations of the dipper and were continued for 4 days. On the 14th day, all subjects were conditioned to leverpress and on the following day were allowed to make 100 reinforced responses. On the 16 th day subjects were divided randomly into two groups $C R$ and VR, and began acquisition training. For the CR group, acquisition was under a CRF schedule throughout. For the VR group, acquisition was under a VR-2 reinforcement schedule. All subjects were given 100 leverpresses daily for 10 days. A discrete trial procedure was used and the lever, which required 4 sec to retract and extend fully, was inoperative during retraction.

On the day following completion of acquisition, each group was subdivided randomly, resulting in the following 12 groups $(\mathrm{N}=8)$ :

Groups CR(ECS)4 and VR(ECS)4. On treatment day, subjects in these two groups were fitted with a harness. Fine wire from an ECS source entered the operant chamber from the top, was connected to the harness, and from the harness to microalligator clips which attached to subject's ears. The harness arrangement permitted complete freedom of movement to all parts of the operant chamber. The first leverpress produced a 9-mA footshock of $2-\mathrm{sec}$ duration, delivered through the grid floor, and the lever retracted. The offset of footshock initiated the onset of a 50-mA 500-msec ECS delivered through the ear-clip electrodes. All subjects in these two groups were tested $4 \mathrm{~h}$ after treatment.

Groups CR(SO)4 and VR(SO)4. For subjects in these groups, treatment was the same as for the above two groups except that footshock was not followed by ECS.

Groups CR(ECS)24 and VR(ECS)24. Treatment for subjects in these two groups was identical to that given to the CR(ECS) 4 and VR(ECS)4 groups, except that testing was delayed until $24 \mathrm{~h}$ after treatment.

Groups CR(SO)24 and VR(SO)24. Treatment for subjects in these two groups was identical to that given to groups CR(SO) 4 and $\operatorname{VR}(\mathrm{SO}) 4$ except that testing was delayed until $24 \mathrm{~h}$ after treatment.

Groups CR(ECS)48 and VR(ECS)48. For subjects in these two groups, treatment was identical to the above ECS groups except that testing was delayed until $48 \mathrm{~h}$ after treatment.

Groups CR(SO) 48 and VR(SO)48. For subjects in these two groups, treatment was identical to the above shock only groups except that testing was delayed until $48 \mathrm{~h}$ after treatment.

For all groups, testing consisted of a 10 -min extinction period, occurring either 4,24 , or $48 \mathrm{~h}$ after treatment. The stimulus conditions during extinction were the same as during acquisition except that the dipper was inoperative.

\section{RESULTS AND DISCUSSION}

The total number of responses made by each subject for the 10-min extinction period was recorded and results showed that more responses were made by Group VR(ECS) 48, followed in order by Groups VR(ECS)24, VR(ECS)4， CR(ECS)4， CR(ECS)24， CR(ECS)48, VR(SO)4, VR(SO)48, CR(SO)48, CR(SO)4, VR(SO)24, $\mathrm{CR}(\mathrm{SO}) 24$. These data were subjected to an analysis of variance which showed that the main effects of treatment and schedule were significant beyond the .05 level. The delay main effect and Schedule by Treatment interaction were not significant at the .05 level.

Group comparisons for treatment effects showed the following differences to be significant beyond the .05 level: $\operatorname{VR}(\mathrm{ECS}) 48$ vs VR(SO)48; $\mathrm{CR}(\mathrm{ECS}) 24$ vs $\mathrm{CR}(\mathrm{SO}) 24 ; \quad \mathrm{CR}(\mathrm{ECS}) 4$ vs $\mathrm{CR}(\mathrm{SO}) 4 ; \mathrm{VR}(\mathrm{ECS}) 4$ vs $\mathrm{VR}(\mathrm{SO}) 4$; $\mathrm{VR}(\mathrm{ECS}) 4$ vs VR(SO)24. Group comparisons for schedule effects showed that CR(ECS)48 vs VR(ECS) 48 was significant beyond the .05 level. All other comparisons were not significant at the .05 level. The mean number of responses in extinction for all groups is shown in Table 1.

The finding that subjects tested $24 \mathrm{~h}$ after receiving ECS made significantly more responses than the shock only groups, regardless of reinforcement schedule replicates earlier findings (Young \& Galluscio, 1970) and was expected.

An examination of the data indicates that the mean number of responses decreased slightly but insignificantly for CR(ECS) subjects as the delay period increased, whereas the mean number of responses increased for VR(ECS) subjects. These data are in partial agreement with previous findings (Young \& Galluscio, 1971; Kohlenberg \& Trabasso, 1968; Cooper \& Koppenall, 1964), and suggest that the effects of ECS on response suppression due to aversive stimulation may be transient in nature.

Perhaps the most critical finding of this study is that after a 48-h delay period following ECS, there was spontaneous recovery of the PRE, although VR-trained subjects failed to show a significant PRE when tested either 4 or $24 \mathrm{~h}$ after completion of acquisition and treatment. This finding was primarily due to a gradual increase in the mean number of responses by VR(ECS) subjects as the ECS-testing period was increased from 4 to $48 \mathrm{~h}$.

The present findings, along with those previously cited, suggest that the effect of ECS is dependent in part upon the schedule of reinforcement employed during acquisition training. In the Young and Galluscio (1971) study, there was no spontaneous recovery of the PRE for FR-trained subjects tested 10 days after ECS 
treatment. If the PRE is caused by some type of conditioned emotional response such as frustration (Amsel, 1958), it could be that ECS interferes in some way with the conditioned frustrative reaction, resulting in a loss of the PRE. This interference, however, appears to differentially affect VR-and FR-trained subjects. The combined findings suggest that reinforcement schedule is an important variable in determinining the effects of ECS, although it is not clear that a memory consolidation hypothesis would suggest it.

\section{REFERENCES}

Amsel, A. The role of frustrative nonreward in noncontinuous reward situations. Psychological Bulletin, 1958, 55, 102-119. Cooper, R. M., \& Koppenaal, R. J. Suppression and recovery of a one-triai avoidance response after a single ECS. Psychonomic Science, 1964, 1, 303-304.

Kohlenberg, R., \& Trabasso, T. Recovery of a conditioned emotional response after one or two electroconvulsive shocks. Journal of Comparative and Physiological Psychology, 1968, 65, 270-273.

Lewis, D. J. Sources of experimental amnesia. Psychological Review, 1969, 76, 461-472.

McGaugh, J L. Time-dependent processes in memory storage. Science, 1966, 153, 1351-1358.

Young, A. G., \& Galluscio, E. H. Effect of ECS on one-trial learning following continuous and partial reinforcement training. Psychonomic Science, 1970, 21, 43-44.

Young, A. G., \& Galluscio, E. H. Recovery from ECS-produced amnesia. Psychonomic Science, 1971, 22, 149-151.

(Received for publication November 13, 1974.)

\title{
Convergence and stereoscopic depth shifts produced by interocular delays in stimulation
}

\author{
EUGENE R. WIST \\ Whitely Psychology Laboratories, Franklin and Marshall College \\ Lancaster, Pennsylvania 17604
}

\begin{abstract}
The possibility that changes in perceived stereoscopic depth resulting from interocular delays in stimulation are due to small increases in the convergence of the eyes during the delay intervals was examined. An indirect method involving the measurement of fixation disparity was used to indicate possible convergence movements. It was found that the magnitude of the shift in perceived stereoscopic depth increased with increasing interocular delay interval as did the magnitude of convergence, but that the magnitude of the former was twice that of the latter. Therefore, the possibility that convergence shifts were responsible for depth shifts was ruled out. The alternative hypothesis that such depth shifts are the result of a central equivalency of binocular disparity and temporal disparity is consequently still tenable.
\end{abstract}

Previous studies (Wist, 1968; Wist, 1970; Wist \& Gogel, 1966) have shown that a temporal disparity in stimulation of the two eyes has an effect on perceived stereoscopic depth which is equivalent to that produced by an increase in binocular (spatial) disparity. This was demonstrated by presenting a luminous fixation stimulus which was binocularly viewed and continuously

This research was supported by Grant EY00268 from the National Eye Research Institute of the U.S. Public Health Service. This report was written while the author was a "Senior U.S. Scientist Awardee of the Alexander von Humboldt Foundation" at the Department of Neurophysiology, University of Freiburg, West Germany. illuminated and a luminous test stimulus which was dichoptically viewed and intermittently illuminated. The left eye was exposed to a 5-msec presentation of the test stimulus and after a variable interocular delay interval (IDI) of 0 to $96 \mathrm{msec}$, the right eye was exposed to the test stimulus for the same exposure duration. The binocular disparity between the test and fixation stimuli was set at 0 so that when IDI was 0 , the two stimuli appeared equidistant. It was found that if the IDI exceeded some $32 \mathrm{msec}$, the perceived depth interval between test and fixation stimuli changed, with the test stimulus appearing increasingly behind the fixation 